April 1999

IFP-772-UNC

\title{
Mersenne Primes, Polygonal Anomalies and String Theories Classification
}

\author{
Paul H. Frampton ${ }^{(a)}$ and Thomas W. Kephart ${ }^{(b)}$ \\ (a)Department of Physics and Astronomy, \\ University of North Carolina, Chapel Hill, NC 27599. \\ (b)Department of Physics and Astronomy, \\ Vanderbilt University, Nashville, TN 37325.
}

\begin{abstract}
It is pointed out that the Mersenne primes $M_{p}=\left(2^{p}-1\right)$ and associated perfect numbers $\mathcal{M}_{p}=2^{p-1} M_{p}$ play a significant role in string theory; this observation may suggest a classification of consistent string theories.
\end{abstract}

Typeset using REVTEX 
Anomalies and their avoidance have provided a guidepost in constraining viable particle physics theories. From the standard model to superstrings, the importance of finding models where the concelation of local and global anomalies that spoil local invariance properties of theories, and hence render them inconsistent, cannot be overestimated. The fact that anomalous thories can be dropped from contention has made progress toward the true theory of elementary particles proceed at an enormously accelerated rate. Here we take up a systematic search, informed by previous results and as yet partially understood connections to number theory, for theories free of leading gauge anomalies in higher dimensions. We will find new cases and be able to place previous results in perspective.

In number theory a very important role is played by the Mersenne primes $M_{p}$ based on the formula

$$
M_{p}=2^{p}-1
$$

where $p$ is a prime number. $M_{p}$ is sometimes itself a prime number. The first 33 such Mersenne primes correspond [1] to prime numbers below one million:

$$
\begin{aligned}
p= & 2,3,5,7,13,17,19,31,61,89,107,127,521,607,1279,2203,2281, \\
& 3217,4253,4423,9689,9941,11213,19937,21701,23209,44497, \\
& 86243,110503,132049,216091,756839,859433 .
\end{aligned}
$$

As a comparison to this remarkable sequence of the first 33 Mersenne primes, there are altogether 78498 primes below one million so that Eq.(1), although an invaluable source of large prime numbers, far more often generates a composite number than a prime.

On the occasion that Eq.(1) does generate a prime, an immediate derivative thereof is the perfect number which we shall designate $\mathcal{M}_{p}$ given by $\mathcal{M}_{p}=2^{p-1} M_{p}$. It is straightforward and pleasurable to prove in general that $\mathcal{M}_{p}$ is perfect, defined as $\mathcal{M}_{p}$ equalling the sum of all of its divisors. For example, $\mathcal{M}_{2}=6=1+2+3, \mathcal{M}_{3}=28=1+2+4+7+14$, and so on. The $\mathcal{M}_{p}$ are the only even perfect numbers; it is unknown if there is an odd perfect number but if there is one it is known [4] that it is larger than $10^{300}$. 
In the present Letter, we shall associate the perfect numbers derived from Mersenne primes with the polygonal anomalies whose cancellation underlies the successful string theories.

For example, heterotic and type-I superstrings in ten dimensions are selected to have gauge groups $O(32)$ and $E(8) \times E(8)$ on the basis of anomaly cancellation of the hexagon anomaly [5 8]. Equivalently, these two superstrings correspond to the only self-dual lattices in 16 dimensions: $\Gamma_{8} \oplus \Gamma_{8}$ and $\Gamma_{16}$ [9]. The dimension of these two acceptable gauge groups in $d=10$ is $\operatorname{dim}(G)=496=\mathcal{M}_{5}$, indeed a perfect number of the Mersenne sequence. Further motivation in low dimensions for consideration of the perfect number comes from [10] $\mathcal{M}_{3}$ the $S O(8)$ and $G_{2} \times G_{2}$ supergravities in 6 dimensions for $\mathcal{M}_{3}$, from noting that $0(4)$ and $S U(2) \times S U(2)$ are anomaly free in four dimensions for $\mathcal{M}_{2}$ and from the existence of an $\mathcal{N}=2$ world sheet supersymmetric string theory in 2 dimensions [11] with gauge group $S O(2) \sim U(1)$ for $\mathcal{M}_{1}$.

The appropriate polygon for spacetime dimension $d$ is the $l$-agon where $l=\left(\frac{d}{2}+1\right)$.

One way to discover the significance of $M_{p}$ and $\mathcal{M}_{p}$ in string theory is to recognize that the leading $l$-agon anomaly for a k-rank tensor of $S U(N)$ or $O(N)$ is given [5.7] by a generalized Eulerian number ( the Eulerian numbers are $A_{N}(N, k)$ )

$$
A_{l}(N, k)=\sum_{p=1}^{k-1}(-1)^{k-p-1}(k-p)^{l-1}\left(\begin{array}{l}
N \\
p
\end{array}\right)
$$

Our purpose here is to investigate the space-time dimensions corresponding to the Mersenne primes $D=2 p$ for gauge group irreps with vanishing leading gauge anomalies. One could then cancel the nonleading anomalies in the Green-Schwarz mechanism to generate a candidate string theory or supergravity (a complete theory must also avoid all local gravitational and global anomalies).

Since all the primes except 2 are odd, the Mersenne prime dimensions (MPDs) are $D=4 n+2$, where $n$ is an integer except for the special case $D=4$. A thorough investigation of the MPDs returns the following for $D$ between 4 and 26 and certain higher values: 
$D=4$ is well studied in the literature, to which we refer the reader [13.

$D=6(p=3)$ : Expressing antisymmetric tensor irreps by $[1]^{k}$ we again find anomaly freedom for the second rank antisymmetric tensor $k=2$ when $N=8$ for gauge groups $S U(8)$ or $S O(8)$. For $S U(N)$ one expects the conjugate solution $[1]^{N-k}=[1]^{8-2}=[1]^{6}$, which is nothing new, but due to its low order, the anomaly polynomial factorizes at $[1]^{6}$ to $(n-6)(N-27)$, implying a nonvanishing anomaly of $[1]^{6}$ for $S U(27)$ [and for $\left.S O(27)\right]$. This in turn implies a $[1]^{N-k}=[1]^{27-6}=[1]^{21}$ solution, which one finds at $N=98$. This sequence continues, (see Table 1) (We remind the reader that for $S U(N),[1]^{N-k}$ and $[1]^{k}$ are complex irreps except when $k=N / 2$ and $N$ is even where $[1]^{N / 2}$ is a real irrep. For $S O(N),[1]^{k}$ is real for $k<N / 2$. When $k=N / 2,[1]^{k}$ splits. The components are real if $N / 2$ is even and they are a complex conjuate pair if $N / 2$ is odd.There are added subtlities for $S O(8)$ because of triality [13].)

$D=10$. As with $D=6$ we find a $[1]^{2}$ solution when $N=2 p=32$. There are two further solutions, up to conjugation $k=4$ with $N=12$, and $k=10$ with $N=32$, and no others with $k \leq 40$. (In what follows we do a study of all cases out to $k=40$, unless noted otherwise.)

$D=14$ : The only solution is $k=2$ for $N=128$.

The case where $p=13$ deserves special consideration, since it corresponds to 26 dimensions, and a $26 D$ theory with $S O\left(2^{13}\right)=S O(8192)$ has indeed already been considered in the literature [14 [16]. In [14], the single dilaton emission amplitude from a disk world sheet was calculated and used in a proof that the total dilaton emission amplitude (from the projective plane plus the disk [17]) at this order vanishes in $26 D$ for $S O(8192)$. Furthermore, it has been shown [15] that the one-loop divergences are avoided by $S O(8192)$ open strings in $26 D$. A general understanding has been provided [16] of the Chan-Paton factors for $S O\left(2^{D / 2}\right)$ in terms of $\mathrm{D}$ added fermionic variables at the ends of open strings, and this is useful input into developing the partition function for the $S O(8192)$ open string [16]. 
Likewise, the only solution is $k=2$ for $N=2^{p}$, with $D=2 p=$ $34,38,62,178,214,254,1042,1214,2558$ and 4406, where we have searched through $k=40$ except for $D=1042$, where $k \leq 24, D=2558$ where $k \leq 10$, and $D=4406$ where $k \leq 8$.

For the sake of completeness, we have also studied the remaining even dimensions below $D=26$, with no Mersenne prime correspondence. As before, $k=2$ with $N=2^{p}$ is always a solution, and when $D=4 n$ ( $n$ integer),

$k=N / 2$ is also a solution as expected since it is real. (Recall that real representations have no anomalies in $D=4 n$ dimensions, but do in $D=4 n+2$, therefore anomaly freedom for $[1]^{k}$ irreps is trivial in $D=4 n$ for $S O$ groups, but not for $S U$ groups.)

$D=8$ : We find the usual $k=2$ and $k=N / 2$ solutions, plus two more sequences, one starting with $k=2, D=16$, and the other with $k=3, D=27$ (see Table 1 ).

$D=12$ : Has only $k=2$ and $k=N / 2$ solutions.

$D=16:$ Has the usual $k=2$ and $k=N / 2$ solutions, plus at $k=3$ with $N=27$ and also at $k=3$ with $N=486$.

$D=18,20,22$ and 24 have nothing new beyond the usual solutions, of $k=2$ and $k=$ $N-2$ for $S U\left(N^{p}\right)$ with $p=D / 2$, and for $D=4 n$ the trivial case of the real representation $k=N / 2$ for any $S U(N)$.

This completes the classification.

Returning to $D=8$, the $[1]^{3}=2925$ of $S U(27)$ or $S O(27)$ is anomaly free, but also the $[1]^{3}$ of $E_{6}$ is a 2925 under the decomposition $S U(27) \longrightarrow E_{6}$, where $27 \longrightarrow 27$. Since the generalized Casimir invariants of $E_{6}$ are of $\operatorname{rank} 2,5,6,8,9$, and 12 , leading anomalies are expected at $D=2,8,20,14,16$, and 22. [18]. However, the 2925 is an exception since it is real.

In $D=6$ no leading $E_{6}$ anomalies are expected, and we find the $k=6, N=27$ result corresponding to the $[1]^{6}=296010$ irrep of $S U(27)$ or $S O(27)$ is reducible in $E_{6}$.

In $D=16$ for $k=3$ and $N=27$, leading $E_{6}$ anomalies are voided by the 2925 . 
The higher $N$ exotic solutions have no obvious origins in exceptional groups.

Our findings are also summarized in Table 1.

Given the well established significance of $\mathcal{M}_{5}$ in spacetime dimension $D=10$ for the two heterotic strings $S O(32)$ and $E_{8} \times E_{8}$ we are led to observe that for $k=2$ (dimensionality $\left.\mathcal{M}_{p}\right)$ of $S O\left(2^{p}\right)$ in spacetime dimensions $D=2 p$ for any of the Mersenne primes, as well as the other particular cases listed in our Table 1, the leading polygonal anomaly $((p+1)$-agon is cancelled. With the possibility that the non-leading anomalies are also cancelled, we are naturally led to speculate that there exist consistent string theories, beyond those presently established, in the spacetime dimensions and involving the particular gauge groups to which we have been led.

This speculation, if verified, will provide one more link between number theory, particularly the Mersenne primes, and string theory.

We thank John Schwarz for drawing our attention to ref. [15]. TWK thanks PHF and the Department of Physics and Astronomy at UNC Chapel Hill for their hospitality while this work was in progress. This work was supported in part by the U.S. Department of Energy under Grants No. DE-FG02-97ER41036 and .DE-FG-5-85ER40226 
Table 1. Solutions of vanishing leading polygonal gauge anomalies. Given a $k$ and $N$, we can find the next value of $k$ (say $k^{*}$ ) from $(N-k)$ but the next $\mathrm{N}$ value (say $N^{*}$ ) corresponding to $k^{*}$ requires a calculation. We have been able to do this calculation up to where a "?" appears.

$\underline{\text { Notes: }}$

${ }_{N} C_{M}$ is the binomial coefficient ${ }_{N} C_{M}=N ! /(M !(N-M) !)$.

$\dagger$ denotes $D=2 p$ where $p$ is a Mersenne prime $M_{p}$ (c.f. Eq.(2)).

$\sharp$ denotes the perfect number $\mathcal{M}_{p}=2^{p-1} M_{p}$. 
Table 1

\begin{tabular}{|c|c|c|c|}
\hline Spacetime dimension(D) & $\mathrm{N}$ of $\mathrm{SO}(\mathrm{N})$ & $k$ of irrep & dimension of irrep. \\
\hline 4 & see Ref. [13] & see Ref. 13 & see Ref. 13 \\
\hline \multirow[t]{6}{*}{$6 \dagger$} & 8 & 2 & ${ }_{8} C_{2}=\mathcal{M}_{3} \ddagger \ddagger$ \\
\hline & 27 & 6 & ${ }_{27} C_{6}$ \\
\hline & 98 & 21 & ${ }_{98} C_{21}$ \\
\hline & 363 & 77 & ${ }_{363} C_{77}$ \\
\hline & 1352 & 286 & ${ }_{1352} C_{286}$ \\
\hline & $?$ & 1064 & $?$ \\
\hline \multirow[t]{7}{*}{8} & 16 & 2 & ${ }_{16} C_{2}$ \\
\hline & 27 & 3 & ${ }_{27} C_{3}$ \\
\hline & 147 & 14 & ${ }_{147} C_{14}$ \\
\hline & 256 & 24 & ${ }_{256} C_{14}$ \\
\hline & 1444 & 133 & ${ }_{1444} C_{133}$ \\
\hline & $?$ & 232 & $?$ \\
\hline & $?$ & 1311 & $?$ \\
\hline \multirow[t]{3}{*}{$10 \dagger$} & 12 & 4 & ${ }_{12} C_{4}$ \\
\hline & 32 & 2 & ${ }_{32} C_{2}=\mathcal{M}_{5} \ddagger \ddagger$ \\
\hline & 32 & 10 & ${ }_{32} C_{10}$ \\
\hline \multirow[t]{2}{*}{12} & $\mathrm{~N}=$ even & $\frac{N}{2}$ & ${ }_{N} C_{N / 2}$ \\
\hline & $2^{6}$ & 2 & ${ }_{64} C_{2}$ \\
\hline $14 \dagger$ & 128 & 2 & ${ }_{128} C_{2}=\mathcal{M}_{7} \ddagger \ddagger$ \\
\hline \multirow[t]{4}{*}{16} & $\mathrm{~N}=$ even & $\frac{N}{2}$ & ${ }_{N} C_{N / 2}$ \\
\hline & 27 & 3 & ${ }_{27} C_{3}$ \\
\hline & $2^{8}$ & 2 & ${ }_{256} C_{2}$ \\
\hline & 486 & 3 & ${ }_{486} C_{3}$ \\
\hline
\end{tabular}


Table 1(continued)

\begin{tabular}{|c|c|c|c|}
\hline Spacetime dimension(D) & $\mathrm{N}$ of $\mathrm{SO}(\mathrm{N})$ & $k$ of irrep & dimension of irrep. \\
\hline 18 & $2^{9}$ & 2 & ${ }_{512} C_{2}$ \\
\hline \multirow[t]{2}{*}{20} & $\mathrm{~N}=$ even & $\frac{N}{2}$ & ${ }_{N} C_{N / 2}$ \\
\hline & $2^{10}$ & 2 & ${ }_{1024} C_{2}$ \\
\hline $22 \dagger$ & $2^{11}$ & 2 & ${ }_{2048} C_{2}=\mathcal{M}_{11} \ddagger \ddagger$ \\
\hline \multirow[t]{2}{*}{24} & $\mathrm{~N}=$ even & $\frac{N}{2}$ & ${ }_{N} C_{N / 2}$ \\
\hline & $2^{12}$ & 2 & ${ }_{4096} C_{2}$ \\
\hline $26 \dagger$ & $2^{13}$ & 2 & ${ }_{8192} C_{2}=\mathcal{M}_{13} \ddagger \ddagger$ \\
\hline \multirow[t]{2}{*}{$\mathrm{D}=4 \mathrm{n}$} & $\mathrm{N}=$ even & $\frac{N}{2}$ & ${ }_{N} C_{N / 2}$ \\
\hline & $2^{2 n}$ & 2 & ${ }_{4}{ }^{n} C_{2}$ \\
\hline$D=4 n+2$ & $2^{2 n+1}$ & 2 & ${ }_{2}^{2 n+1} C_{2}$ \\
\hline $\mathrm{D}=2 \mathrm{p}(\mathrm{p}=$ Mersenne $) \dagger$ & $2^{p}$ & 2 & $\mathcal{M}_{p} \ddagger \ddagger$ \\
\hline $34 \dagger, 38 \dagger, 62 \dagger, 178 \dagger, 214 \dagger, 254 \dagger$ & $?$ & $?(k>40)$ & $?$ \\
\hline $1042 \dagger$ & $?$ & $?(k>24)$ & $?$ \\
\hline $1214 \dagger$ & $?$ & $?(k>15)$ & $?$ \\
\hline $2558 \dagger$ & $?$ & $?(k>10)$ & $?$ \\
\hline $4406 \dagger$ & $?$ & $?(k>8)$ & $?$ \\
\hline$D=(2 p) \dagger \geq 4562 \dagger$ & $?$ & $?$ & $?$ \\
\hline
\end{tabular}




\section{REFERENCES}

[1] G.H. Hardy and E.M. Wright, An Introduction to the Theory of Numbers. Oxford. Fifth Edition (1979).

[2] Encyclopedic Dictionary of Mathematics. Editor: K.Ito.

MIT Press, Second Edition (1987). Section 297.

[3] A useful source for prime number searches is at the web page: http://www.utm.edu/research/primes

[4] R.P. Brent, G.L. Cohen and H.J.J. te Riele, Math. Comp. 57, 857 (1991).

[5] P.H. Frampton and T.W. Kephart, Phys. Rev. Lett. 50, 1343 (1983).

[6] P.H. Frampton and T.W. Kephart, Phys. Rev. Lett. 50, 1347 (1983).

[7] P.H. Frampton and T.W. Kephart, Phys. Rev. D28, 1010 (1983).

[8] P. K. Townsend and G. Sierra, Nucl. Phys. B222, 493 (1983).

[9] J. P. Serre, A Course in Arithmetic. Springer (1985).

[10] R. Holman and T. W. Kephart, Phys. Lett. B 1999 to appear.

[11] M. Ademollo, it et al., Nucl. Phys. B111, 77(1976).

[12] M.B. Green and J.H. Schwarz, Phys. Lett. 149B, 117 (1984).

[13] R. Slansky, Phys. Reports, 79, 1 (1981).

[14] M. R. Douglas and B. Grinstein, Phys. Lett. 183B, 52 (1987).

[15] S. Weinberg, Phys. Lett. 187B, 278 (1987).

[16] M. Bianchi and A. Sagnotti, Phys. Lett. 211B, 407 (1988).

[17] B. Grinstein and M. B. Wise, Phys. Rev. D35, 655 (1987).

[18] T.W. Kephart and M.T. Vaughn, Ann. Phys. 145, 162 (1983). 